\title{
The Blackburn handover - an innovative development to support patient, trainee, staff and organisational safety
}

\author{
Authors: Junaid Aamir, Suzanne Gawne and Damien Lynch
}

\begin{abstract}
Background
High-quality medical handover $(\mathrm{MH})$ of patients is essential in helping to deliver effective care. Current literature encourages hospitals to adopt formalised handover structures to enhance patient safety and team working. ${ }^{1,2}$ Furthermore, shift handovers can be utilised as a learning tool for the medical team. East Lancashire Hospitals NHS Trust (ELHT) was placed in special measures by Health Education England North West (HEENW) for its postgraduate medical training. The lack of a formal MH system was highlighted as a particular area of concern. It was not effective and had no educational value. There was a need to develop and implement an effective $\mathrm{MH}$ to support patient, trainee, staff and organisational safety.
\end{abstract}

\section{Materials and methods}

An operational and educational lead was appointed to oversee $\mathrm{MH}$ development and implementation. The approach was 'bottom up' with multiprofessional stakeholder engagement primarily from trainees.

The MH at ELHT was redesigned and implemented. It was designed to be multiprofessional, timely, pragmatic, educational, meaningful and pastoral. It aimed to:

$>$ engage learners to produce an educational environment facilitated by a consultant

> develop leadership, team working, and promote pastoral support for attendees

> create a culture to support the raising of concerns with a nonblame professional approach

$>$ address organisational and safety issues

> disseminate $\mathrm{MH}$ information to a wider audience to encourage organisational learning and reach those able to facilitate prompt change

> fulfil the Royal College of Physicians (RCP) criteria for $\mathrm{MH}$.

A set agenda comprised sections to address patient handover, staffing and system issues, raising concerns, team wellbeing and an educational component. Chaired by an ST3+ to develop leadership skills, a consultant facilitator was present to encourage reflection in a supportive environment.

Authors: East Lancashire Hospitals Trust
Outcome measures comprised ELHT trainee, General Medical Council (GMC) National Trainee Survey and HEENW feedback.

\section{Results and discussion}

Trainee response to an online feedback questionnaire demonstrated $>90 \%$ approval (agree/strongly agree) with regard to trainee education, raising concerns and supporting patient safety. Qualitative responses included the following:

> 'Very responsive to feedback, timely responses (usually same day), excellent, first time I have worked in a trust that has been this responsive to trainees/on-call issues.'

> 'Allows urgent patient safety issues from wards to be rapidly addressed.'

> 'Raising concerns is welcomed in the meeting and they are taken on board. They are also actioned.'

> 'Professional but supportive atmosphere - feel able as an FY1 to ask if unsure or hand over perhaps less important issues without scorn!'

Trainees have stated that working relationships within the trust have improved as a result of feedback they have received from the $\mathrm{MH}$.

Following the new MH introduction the GMC National Trainee Survey demonstrated a year-on-year increase in trainee satisfaction in a number of domains, particularly, 'handover', 'educational supervision', 'levels of feedback' and 'supportive environment'. Reported as 'Excellent' by junior doctor advisory team (2016), HEENW (2018) described it as an exemplar with regard to the level of education and support offered to trainees. The MH stakeholders comprise all trainees and senior colleagues working medical shifts. Handover also involves nursing and allied health professionals, clinical support and diagnostic services including information technology, radiology and laboratory services, and middle and senior trust management. The format is under continual development based on feedback from all members of the workforce. This is a truly multiprofessional activity.

The $\mathrm{MH}$ lasts an average of 14 minutes, in keeping with a timely approach allowing outgoing teams to leave promptly. A pastoral section is dedicated to trainee welfare and wellbeing including appreciative inquiry and ensuring rest facilities are available following a shift. Feedback is provided at the end of the $\mathrm{MH}$ by the attending consultant to support trainee development. E-teaching on clinical issues occurs following the $\mathrm{MH}$ and challenging clinical 
or ethical incidents experienced by trainees highlighted at the $\mathrm{MH}$ are used as topics for discussion at the medical grand round attended by different professional groups to support broader learning. Trainees learn the importance of teamworking, leadership and professionalism in a supervised environment. A seating plan supports the $\mathrm{MH}$ effectiveness.

The identification of information technology and medical systems issues throughout the shifts and prompt contact with relevant departments has allowed problems to be rectified as soon as possible. This has resulted in minimal delays with hospital systems and improved levels of patient care.

There have been numerous improvements in patient safety following concerns raised at the $\mathrm{MH}$ including clinical pathway development, out of hour emergency communications and effective patient handover. The MH also acts as a platform for quality improvement projects.

\section{Conclusion}

The 'Blackburn handover' has helped promote patient, trainee, staff and organisational safety at ELHT and supports the broader development of trainees. There is potential for it to have a wider application across healthcare organisations.

\section{References}

1 Pucher PH, Johnston MJ, Aggarwal R, Arora S, Darzi A. Effectiveness of interventions to improve patient handover in surgery: A systematic review. Surgery 2015;158:85-95.

2 Segall N, Bonifacio AS, Schroeder RA et al. Patient Safety Center of Inquiry. Can we make postoperative patient handovers safer? A systematic review of the literature. Anesthesia and Analgesia 2012;115:102-15. 